\title{
Novel microstructured fibres for supercontinuum generation
}

\author{
X. Jiang ${ }^{1, *}$, N.Y. Joly ${ }^{2,1}$, F. Babic ${ }^{1}$, R. Sopalla ${ }^{1}$, R. Song ${ }^{3}$, J. Lousteau ${ }^{4,5}$, D. Milanese ${ }^{5}$, \\ J.C. Travers ${ }^{1}$ and P. St.J. Russell ${ }^{1,2}$ \\ ${ }^{1}$ Max-Planck-Institute for the Science of Light and ${ }^{2}$ Department of Physics, University of Erlangen-Nuremberg, \\ Guenther-Scharowsky-Strasse 1, 91058 Erlangen, Germany \\ ${ }^{3}$ College of Optoelectronic Science and Engineering, National University of Defense Technology, 410073 Changsha, China \\ ${ }^{4}$ Optoelectronics Research Centre, University of Southampton, Highfield, Southampton, SO17 1BJ, United Kingdom \\ ${ }^{5}$ Institute of Materials Physics and Engineering, DISAT-Politecnico di Torino, Corso Duca degli Abruzzi 24, 10129 Torino, Italy \\ xin.jiang@mpl.mpg.de
}

\begin{abstract}
We report recent progress on the fabrication of photonic crystal fibre from ZBLAN and tellurite glasses and their application to generating broadband supercontinua.

OCIS codes: 060.5295 Photonic crystal fiber; 060.2280 Fiber design and fabrication; 320.6629 Supercontinuum generation.
\end{abstract}

Soft glasses such as fluorides, chalcogenides and heavy-metal oxides have attracted great interest because of their wide windows of transparency extending into the deep ultraviolet (UV) and mid-infrared (mid-IR) spectral regions [1, 2]. Among them, $\mathrm{TeO}_{2}$-based tellurite and $\mathrm{ZrF}_{4}$-based $\mathrm{ZBLAN}$ fluoride glasses have been intensively investigated for supercontinuum (SC) generation. There are several reasons for this. For example, the good thermal stability and chemical resistance of tellurite glasses means that they can be easily melted and moulded into the required shape. In addition, these glasses possess very high linear and nonlinear refractive indices and if properly synthesized, offer a transmission window that can be extended to $6 \mu \mathrm{m}$, suitable for mid-IR SC generation in sub-centimetre fibre lengths [3]. On the other hand, fluoride glasses have extremely low water content and provide low absorption over the entire transparency window from the deep ultraviolet $(200 \mathrm{~nm})$ to the mid-infrared $(8 \mu \mathrm{m})$, making it ideal for the generation of multi-octave-wide supercontinua. Previous work on supercontinuum generation in ZBLAN fluoride glass has however focused on step-index fibres in the spectral range from the visible to the mid-IR [4-6], except for a recently published paper [7] in which SC spectra down to a record $200 \mathrm{~nm}$ in the deep-UV was generated inside a solid-core photonic crystal fibre (PCF). In both tellurite and ZBLAN PCFs, the dispersion can be tailored with proper fibre design. Drawing tellurite or ZBLAN glass PCFs with complicated cladding structures is extreme challenging due to the much more rapid change in glass viscosity with temperature, which means that the drawing temperature must be precisely controlled within a narrow range (less than $30^{\circ} \mathrm{C}$ for tellurite and $10^{\circ} \mathrm{C}$ for ZBLAN) [1,2] - much more challenging than in fused silica $\left(\sim 300^{\circ} \mathrm{C}\right)$. Another challenge is thermal instability, which can cause glass devitrification in fibre preforms during heating. Here we introduce an improved "stack-and-draw" procedure for soft glasses [8], that enables us to fabricate tellurite and ZBLAN PCFs with nearly perfect structures.

(a)
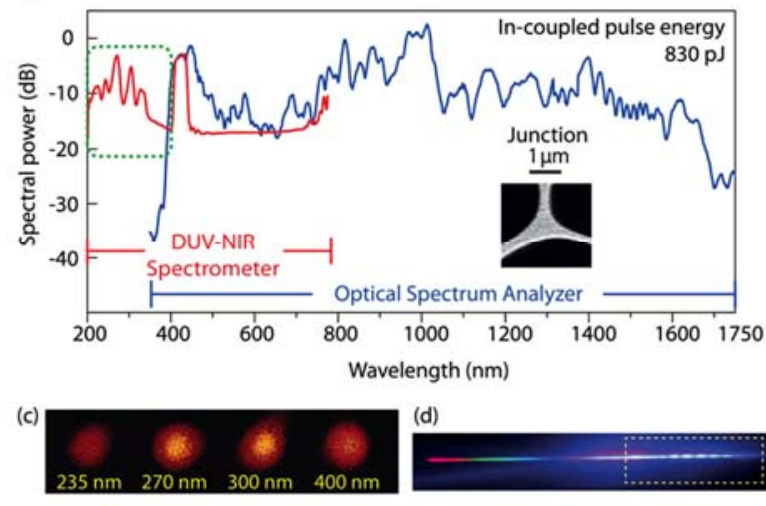

\section{(d)}

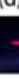

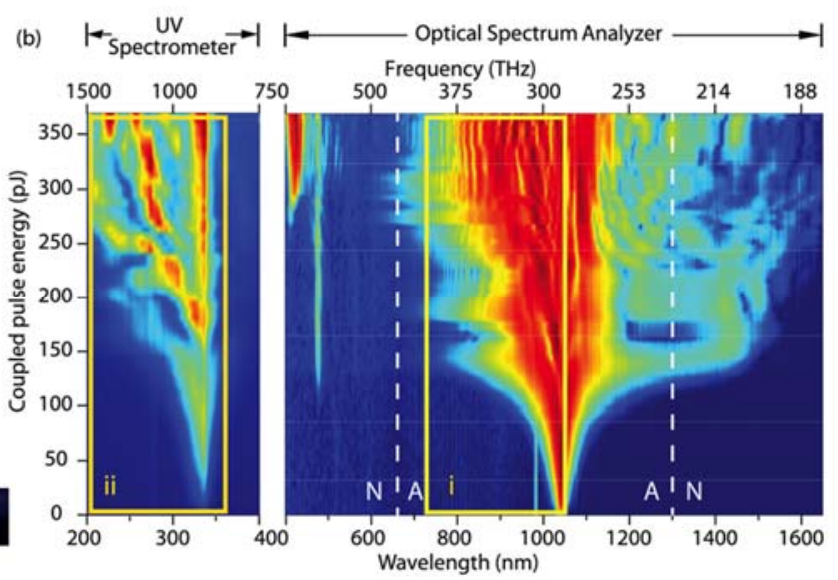

Fig. 1 (a) SC spectrum generated in a cladding junction for an in-coupled pump energy of $830 \mathrm{pJ}$. The spectrum extends from the deep-UV $(200 \mathrm{~nm})$ to the infrared $(1.75 \mu \mathrm{m})$; (b) Energy dependence of UV and visible-near-IR spectra generated in the same junction, measured for in-coupled pulse energies from 3 to $362 \mathrm{pJ}$; (c) Near-field mode profiles measured with different bandpass filters in the UV region; (d) A spatially dispersed spectrum shows a bright deep UV band (within the dashed box).

One of our highly nonlinear solid-core ZBLAN PCFs was used for SC generation, pumped by fs lasers at 800 and $1042 \mathrm{~nm}$. As discussed in [7], one of the interstitial junctions in the cladding was chosen for SC generation due to its 
suitable dispersion profile. When launching $1042 \mathrm{~nm}$ fs pulses into this junction, a SC spanning more than three octaves (200 to $1750 \mathrm{~nm}$ ) was generated in the fundamental mode in a $4 \mathrm{~cm}$ length of the PCF, at an in-coupled pulse energy of only $830 \mathrm{pJ}$ (Fig. 1a). The generated spectra, lifetime measurements, theoretical calculations, and other related results are all discussed in [7] (Fig. 1). The results also give clear evidence that third harmonic generation (THG) may play an important role in the origin of deep-UV emission, even at very low pump energies (Fig. 1b). With increasing pump energy, the light initially generated shifts continuously to the blue towards $200 \mathrm{~nm}$. Although the numerical simulations (not shown here) clearly show some UV light generation, the brightness and multi-peak spectral structure of the UV SC band are not reproduced. The non-reproducibility we attribute to a lack of knowledge of the material properties in the UV region, especially the nonlinear coefficients and the dispersion of ZBLAN.
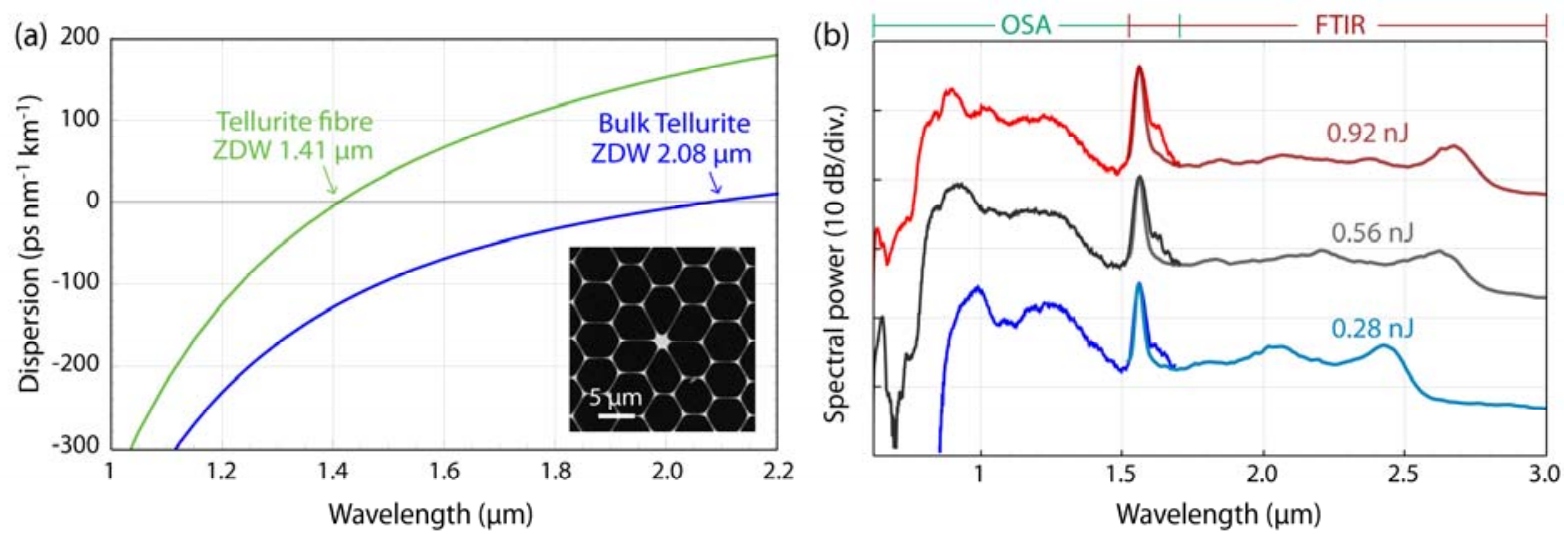

In a second experiment, we launched $150 \mathrm{fs}$ pulses at $1550 \mathrm{~nm}$ into a $3.2 \mathrm{~cm}$ length of highly nonlinear tellurite PCF. The core diameter of the fibre was $\sim 2.4 \mu \mathrm{m}$, as estimated from the SEM in Fig. 2a. The dispersion profiles of the bulk glass and fibre are plotted in the same figure, showing zero dispersion wavelengths at 2.08 and $1.41 \mu \mathrm{m}$. The $1.55 \mu \mathrm{m}$ pump laser wavelength falls within the region of anomalous dispersion. Spectral broadening begins even at very low pulse energies (not shown here). Figure $2 \mathrm{~b}$ shows the measured output spectra for launched pulse energies from 0.28 to $0.92 \mathrm{~nJ}$. In the best case we achieved a $10 \mathrm{~dB}$ flat SC over two-octaves wide, extending from 0.62 to 2.85 $\mu \mathrm{m}$, for a launched pulse energy of $0.92 \mathrm{~nJ}$. The high efficiency of SC generation in such a short fibre piece is only possible by correct tailoring of the dispersion through proper fibre design.

In summary, the talk will review recent work in the fabrication of ZBLAN and tellurite PCFs, and applications to the generation of deep-UV to mid-IR SC in these fibres. Some recent (so far unpublished) results on UV light generation using a pump source at $800 \mathrm{~nm}$ [9] will be directly compared with the results with a $1042 \mathrm{~nm}$ pump laser [7], providing confirmation of the role of THG in the initial stages of spectral broadening to the deep-UV.

\section{References:}

1. M. Cable, J. M. Parker, High-Performance Glasses, Chapman and Hall, 1992.

2. J. S. Sanghera, I. D. Aggarwal, Infrared Fiber Optics, CRC Press, 1998.

3. P. Domachuk, N. A. Wolchover, M. Cronin-Golomb, A. Wang, A. K. George, C. M. B. Cordeiro, J. C. Knight and F. G. Omenetto, "Over $4000 \mathrm{~nm}$ bandwidth of midIR supercontinuum generation in sub-centimeter segments of highly nonlinear tellurite PCFs," Opt. Express, 16, 7161 (2008).

4. C. Xia, M. Kumar, O. P. Kulkarni, M. N. Islam, F. L. Terry, M. J. Freeman, M. Poulain, G. Maze, "Midinfrared supercontinuum generation to $4.5 \mu \mathrm{m}$ in ZBLAN fluoride fibers by nanosecond diode pumping," Opt. Lett. 31, 2553 (2006).

5. G. Qin, X. Yan, C. Kito, M. Liao, C. Chaudhari, T. Suzuki, Y. Ohishi, "Ultrabroadband supercontinuum

generation from ultraviolet to $6.28 \mu \mathrm{m}$ in a fluoride fiber," Appl. Phys. Lett. 95, 161103 (2009).

6. A. M. Heidt, J. H. V. Price, C. Baskiotis, J. S. Feehan, Z. Li, S. U. Alam, D. J. Richardson, "Mid-infrared ZBLAN fiber supercontinuum source using picosecond diodepumping at $2 \mu \mathrm{m}$," Opt. Express 21, 24281 (2013).

7. X. Jiang, N. Y. Joly, M. A. Finger, F. Babic, G. K. L. Wong, J. C. Travers, P. St.J. Russell, "Deep-ultraviolet to mid-infrared supercontinuum generated in solid-core ZBLAN photonic crystal fibre," Nat. Photon. 9, 133 (2015).

8. X. Jiang, T. G. Euser, A. Abdolvand, F. Babic, F. Tani, N. Y. Joly, J. C. Travers, P. St.J. Russell, "Single-mode hollow-core photonic crystal fiber made from soft glass,” Opt. Express 19, 15438 (2011). 